Präv Gesundheitsf 2019 • 14:1-2 https://doi.org/10.1007/s11553-018-0693-7 Online publiziert: 18. Dezember 2018 (c) Springer-Verlag GmbH Germany, part of Springer Nature 2018

CrossMark

\section{Uwe Berger ${ }^{1} \cdot$ Heike Kraußlach ${ }^{2} \cdot$ Bernhard Strauß $^{\prime}$}

${ }^{1}$ Institut für Psychosoziale Medizin und Psychotherapie, Universitätsklinikum Jena, Friedrich-SchillerUniversität Jena, Jena, Deutschland

${ }^{2}$ Fachbereich Betriebswirtschaft, Ernst-Abbe-Hochschule Jena, Jena, Deutschland

\title{
VorteilJena: Vorbeugen durch Teilhabe in der Gesundheitsregion von morgen
}

Gesundheitsförderung und Prävention sind seit Inkrafttreten des Gesetzes zur Stärkung der Gesundheitsförderung und der Prävention (PrävG) am 01.01.2016 [3] deutlicher ins Bewusstsein der Gesundheitsversorgung und Gesundheitsforschung gerückt. Ein wesentlicher Punkt des sog. neuen Präventionsgesetzes ist die finanzielle Stärkung der Setting-bezogenen Gesundheitsförderung. Unter Settings werden hierbei sozialräumliche institutionalisierte Lebenswelten wie Schulen, Unternehmen und Seniorenheime adressiert. Über diese Settings sind im Sinne der OttawaCharta der Weltgesundheitsorganisation (WHO) weite Teile der Bevölkerung für verhaltens- und verhältnispräventive Maßnahmen gut erreichbar [12]. Die Notwendigkeit für solche Bemühungen ergibt sich aus einem seit mehreren Jahrzehnten andauernden Trend der Verschiebung des Krankheitsspektrums in Richtung ernährungs- und bewegungsinduzierter Erkrankungen (allen voran Herz-Kreislauf-Erkrankungen, Diabetes mellitus, aber auch Essstörungen) sowie psychischer Erkrankungen und Symptome, wie Depression, Burnout, chronischem Stress und Angsterkrankungen. Während Herz-Kreislauf-Erkrankungen über $40 \%$ des Sterbegeschehens in Deutschland bestimmen, sind psychische Erkrankungen in steigendem Maße mitverantwortlich für Krankschreibungen und Frühberentungen [9].

Vor diesem Hintergrund schrieb das Bundesministerium für Bildung und Forschung (BMBF) 2012 erneut einen Wettbewerb unter dem Titel „Gesund- heitsregion von Morgen“ aus. Mit der Förderung sollten Modellregionen in Deutschland innovative Maßnahmen der Primärprävention und Gesundheitsförderung im Rahmen eines Forschungsund Praxisverbunds entwickeln und evaluieren (Bundesanzeiger vom 20.09.2012: www.gesundheitsforschung-bmbf.de/ de/4595.php). In der 1. Runde dieser Ausschreibung im Mai 2008 wurden unter dem Titel „Gesundheitsregionen der Zukunft - Fortschritt durch Forschung und Innovation" die Verbundprojekte Psychenet (Hamburg), HIC@RE (Greifswald/Rostock), REGiNA (Stuttgart/Tübingen), Fontane (Nordbrandenburg) und INFOPAT (Mannheim/ Heidelberg) mit insgesamt 37 Mio. $€$ gefördert.

Neben 4 Dienstleistungsregionen Chemnitz+, FISnet (Augsburg/Schwaben), PNO (Präventionsnetz Ortenaukreis) und QuartiersNETZ (Ruhrgebiet) wurde VorteilJena als Gesundheitsregion in einem zweistufigen Verfahren ausgewählt und startete am 1. Oktober 2014 als Verbundprojekt unter dem Förderkennzeichen 01KK1401A-C. Unter dem Leitgedanken „Vorbeugen durch Teilhabe“ wurde in 8 Forschungsprojekten am Universitätsklinikum Jena (UKJ), der Friedrich-Schiller-Universität Jena (FSU) und der Ernst-AbbeHochschule Jena (EAH) der Zusammenhang von sozialer Teilhabe mit den großen Gesundheitsproblemen unserer Zeit praxisnah erforscht (genauer s. [1]).

Soziale Teilhabe hat seit Verabschiedung der Behindertenrechtskonvention der Vereinten Nationen (UN) 2006 [11] verstärkt gesellschaftspolitische Bedeutung erlangt und steht in engem $\mathrm{Zu}$ sammenhang mit den Begriffen Integration und Inklusion (vgl. [7]). Diese beiden Begriffe werden jedoch mittlerweile im öffentlichen Sprachgebrauch in einer stark verkürzten Bedeutung verwendet. So wird Integration oft gleichgesetzt mit dem Zusammenleben von Menschen unterschiedlicher Kulturen. Inklusion wird häufig verkürzt in Bezug auf gleiche Bildungschancen trotz Behinderung oder pädagogischem Förderbedarf definiert (z. B. Teilhabebericht der Bundesregierung [2]). Eigentlich sind mit dem Begriff „Integration“ jedoch viel allgemeiner „Prozesse der Einbindung von Individuen in das normative Gefüge der Gesellschaft" $[4$, S. 60] gemeint sowie die Teilhabe an der Gesellschaft unabhängig von verschiedenen sozialen Kategorien wie Geschlecht, Alter, Herkunft, Religionszugehörigkeit oder Bildung. Bei Inklusion steht über die bloße Einbindung hinaus die gleichberechtigte Teilhabe im Vordergrund, die von der WHO als „Einbezogensein in eine Lebenssituation" [3, S. 16] beschrieben wird. Soziale Teilhabe im inklusiven Sinn ist damit deutlich aktiver und hat unmittelbare Auswirkungen auf den personalen und sozialen Selbstwert und die Identifikation mit gesellschaftlichen Normen und Werten der einzelnen Mitglieder [10]. So verstanden, hat soziale Teilhabe in doppelter Weise gesundheitliche Relevanz. Zum einen steht die Entwicklung der neuen Volkskrankheiten (ernährungs- und bewegungsinduzierte Erkrankungen sowie psychische Erkrankungen) in engem $\mathrm{Zu}$ - 
sammenhang mit sozialen Bedingungen $[3,10]$. Zum anderen hat sich der Selbstwert, bzw. dessen handlungsbezogener Anteil der Selbstwirksamkeit, als zentrale Variable der Veränderung beim gesundheitsrelevanten Handeln erwiesen [8].

„Vorbeugen durch Teilhabe“ steht damit für 2 Kernziele des Verbundprojekts VorteilJena:

1. Förderung sozialer Teilhabe als potenziellem Einflussfaktor auf den Erfolg von Maßnahmen zur Gesundheitsförderung.

2. Stärkung des Selbstwerts (bzw. der Selbstwirksamkeit) als vermittelnder Variable zwischen sozialer Teilhabe und Gesundheitsverhalten.

Diese Ziele wurden in acht Teilprojekten in praxisorientierte empirische Forschung umgesetzt. Zentral war hierbei die Entwicklung, Evaluation und der Transfer von Praxishilfen zur Gesundheitsförderung in den drei Lebenswelten „Gesund Lernen", "Gesund Arbeiten“ und „Gesund Altern" unter stetigem Einbezug der jeweiligen Zielgruppen und Akteure [6].

Die Evaluationsstudien zur Qualitätssicherung der Praxishilfen orientierten sich am dreistufigen Modell der „Society for Prevention Research“ (SPR, [5]). Im 1. Schritt wurden Bedarfsanalysen sowie Machbarkeitsstudien mit vertiefender qualitativer und quantitativer Prozessevaluation durchgeführt (Pilotstudien). Im 2. Schritt folgten kontrollierte Wirkungsstudien. Abschließend wurde im 3. Schritt unter Einbezug der Evaluationsergebnisse und Rückkoppelung der beteiligten Akteure der Transfer für eine bevölkerungsweite Nutzung vorbereitet.

In den folgenden Beiträgen wird die Entwicklung und Evaluation der im Forschungsverbund VorteilJena entstandenen Praxishilfen skizziert. Je nach Umfang der einzelnen Evaluationsstudien innerhalb der Teilprojekte werden insbesondere die, z.T. noch nicht vollständig abgeschlossenen, Wirkungsstudien des 2 . und 3. Schrittes nicht im Detail beschrieben, sondern $\mathrm{zu}$ einem späteren Zeitpunkt an anderer Stelle publiziert. In jedem Fall informieren die Beiträge jedoch über die zum Projektabschluss öffentlich zur Verfügung stehenden Produkte in Form von Setting-baisierten Praxishilfen zur Prävention und Gesundheitsförderung in Schulen, Unternehmen und Seniorenheimen. Weitere Informationen finden sich auf www.vorteiljena.de.

\section{Korrespondenzadresse}

PD Dr. Uwe Berger

Institut für Psychosoziale Medizin und

Psychotherapie, Universitätsklinikum Jena,

Friedrich-Schiller-Universität Jena

Stoystr. 3, 07740 Jena, Deutschland

uwe.berger@med.uni-jena.de

Förderung. Das Verbundprojekt VorteilJena wurde finanziell gefördert vom Bundesministerium für Bildung und Forschung (BMBF; Förderkennzeichen 01KK1401A-C; Laufzeit 10/2014-09/2018).

\section{Einhaltung ethischer Richtlinien}

Interessenkonflikt. U. Berger, H. Kraußlach und B. Strauß geben an, dass kein Interessenkonflikt besteht.

Für die im Rahmen des Verbundprojektes VorteilJena durchgeführten Studien liegt ein positives Votum der Ethikkomission des Universitätsklinikums Jena der Friedrich-Schiller-Universität Jena vor (Bearbeitungsnummern 4417-04/15 und 4477-07/15).

\section{Literatur}

1. Berger U, Kraußlach H, Strauß B (2015) Was zeichnet eine Gesundheitsregion von morgen aus? Das Verbundprojekt VorteilJena - Vorbeugen durch Teilhabe. Psychother Psychosom Med Psychol 65:203

2. Bundesministerium für Arbeit und Soziales (Hrsg) (2013) Teilhabebericht der Bundesregierung übe die Lebenslagen von Menschen mit Beeinträchtigungen. BMAS, Bonn

3. Bundesrat (2015) Gesetz zur Stärkung der Gesundheitsförderung und der Prävention (PrävG). Bundesgesetzesblatt Teil I31:1368-1379

4. Degener T, Mogge-Grotjahn H (2012) „All inclusive"? Annäherungen an ein interdisziplinäres Verständnis von Inklusion. In: Balz HJ, Benz B, Kuhlmann C (Hrsg) Soziale Inklusion. Grundlagen, Strategien und Projekte in der Sozialen Arbeit. Springer VS, Wiesbaden, S59-77

5. Gottfredson DC, Cook TD, Gardner FEM et al (2015) Standards of evidence for efficacy, effectiveness, and scale-up research in prevention science: next generation. Prev Sci 16:893-926

6. Gläser A, Berger U (2016) Integrative und inklusive Ansätze in der Forschung. Psychother Psychosom Med Psychol 66:501-502

7. Kirschner H, Berger U (2015) Was ist Inklusion? Psychother Psychosom Med Psychol 65:445-446

8. Knoll N, Scholz U, Rieckmann N (2011) Einführung in die Gesundheitspsychologie. Ernst Reinhardt, München

9. Robert-Koch-Institut (Hrsg) (2016) Gesundheit in Deutschland - die wichtigsten Entwicklungen.
Gesundheitsberichterstattung des Bundes. Gemeinsam getragen von RKI und Destatis. RKI, Berlin

10. Tajfel H, Turner JC (1986) An integrative theory of intergroup conflict. In: Austin WG, Worchel S (Hrsg) The social psychology of intergroup relations. Brooks, Cole, Monterey, S 33-47

11. Vereinte Nationen (2006) Übereinkommen über die Rechte von Menschen mit Behinderungen. https://www.behindertenrechtskonvention. info/uebereinkommen-ueber-die-rechte-vonmenschen-mit-behinderungen-3101/. Zugegriffen: 14. März 2018

12. Weltgesundheitsorganisation (1986) OttawaCharta zur Gesundheitsförderung. http://www. euro.who.int/de/publications/policy-documents/ ottawa-charter-for-health-promotion,-1986.Zugegriffen: 14. März 2018 